\title{
Getting it right - 3D Cell cultures for the assessment of photosensitizers for photodynamic therapy (PDT)
}

\section{Mathias O. Senge, ${ }^{a^{*}}$ Shelley Stafford ${ }^{\mathrm{b}}$}

* Author for correspondence.

${ }^{a}$ Medicinal Chemistry, Institute of Molecular Medicine, Trinity Centre for Health Sciences, Trinity College Dublin, St James's Hospital, Dublin 8, Ireland

${ }^{b}$ School of Chemistry, SFI Tetrapyrrole Laboratory, Trinity Biomedical Sciences Institute, 152-160 Pearse Street, Trinity College Dublin, The University of Dublin, Dublin 2, Ireland

Tel.: +35318968537

Fax: +35318968536

E-mail: sengem@tcd.ie

Core quote "Cancer drug screening is rapidly moving towards the use of 3D cell models. The added complexity of PDT action makes this a conditio sine qua non for the screening of new photosensitizers."

Keywords: photodynamic therapy $\boldsymbol{\|}$ in vitro models $\boldsymbol{\|}$ photosensitizers $\boldsymbol{\|}$ 3D cell cultures photomedicine a drug development

The quest for the treatment of malignant diseases is ongoing. Classic modalities based on chemo- and radiotherapy and surgery are now supplanted by or used in conjunction with photodynamic therapy (PDT) [1]. PDT involves on the administration of a photosensitizing dye and its accumulation in the affected tissue. After illumination of the respective body part with light (typically low intensity lasers), the photosensitizer transfers its excitation energy to triplet oxygen (the 'regular' form of oxygen in the body) to yield singlet oxygen and other reactive oxygen species (ROS). ROS are highly reactive and cytotoxic, resulting in cell death via apoptosis, necrosis, or autophagy [2]. PDT can be very effective for treating cancers and has found widespread use in dermatology - e.g., as PUVA treatment for actinic keratosis - and is now approved for treating cancer of the esophagus, non-small cell lung cancer and certain types of head \& neck cancers or dysplasias. Prime examples for photosensitizers are porphyrins, chlorophyll derivatives, $\delta$-aminolevulinic acid (precursor for protoporphyrin), simple dyes such as psoralen, and others [3].

Yet, despite its promise, widespread clinical use of PDT is only slowly emerging; in part the result of drawbacks of existing drugs and the slow entry of new drugs into the developmental pipeline. Photosensitizers are often classified as $1^{\text {st }}, 2^{\text {nd }}$ and $3^{\text {rd }}$ generation drugs, with Photofrin being the classic $1^{\text {st }}$ generation PDT drug. This was followed by improvements of the photophysical and pharmacological properties in $2^{\text {nd }}$ generation molecules such as Levulan, Temoporfin or Verteporfin [2]. Currently, a significant number of similar drugs are in preclinical development. Contemporary studies on $3^{\text {rd }}$ generation agents focus on improved targeting, either via bioconjugate strategies (e.g., carbohydrate or antibody appended porphyrins), as nanoformulations or active transport systems $[3,4]$.

An optimum photosensitizer should have a high singlet oxygen quantum yield, not undergo photobleaching, be non-toxic and stable in the dark, water soluble, and be 
selectively retained in high concentrations in the target tissue and readily clear from the body in order to avoid continued photosensitivity. Through advances in the synthetic chemistry of porphyrins and related compounds, chemists can design and synthesize 'optimized' photosensitizers with a high level of sophistication [3]. However, this is no guarantee for their in vitro and in vivo efficacy. Parameters such as tissues clearance, photosensitivity, (dark) phototoxicity, pain and relative enrichment in target tissue are difficult to predict and can only be assessed through cell biological studies. Typically, new photosensitizing agents are evaluated in vitro by investigating the cell viability in simple "2D" cell cultures and comparing the results from dark and illuminated samples.

As for any other drug development such in vitro cytotoxicity tests can only give an indication for the in vivo pharmacology and the ultimate utility of a lead compound. The different levels of complexity still results in many of the in vitro leads failing at the in vivo level, with only $5 \%$ of anti-cancer drugs making it from in vitro studies to the clinic, illustrating how the success of a compound in vitro does not necessarily translate to in vivo studies [5]. In terms of cancer treatment several critical aspects of PDT cannot be modeled at all in standard in vitro cell test. In addition to direct cell killing, a significant part of the "PDT effect" are tissue related, e.g., damage of the microvasculature via anti-angiogenesis and anti-tumor immune responses. Additionally, PDT relies on the availability of oxygen in the target tissue and thus tumor oxygenation and hypoxia impact the overall treatment effect [2].

Clearly, standard 2D monolayer cell cultures do not accurately recreate the tumor environment. Next to obvious features such as the absence of blood vessels and different oxygenation states, cell motility in tissue, many other issues limit the predictive utility of 2D cell cultures. Cells growing in monolayer are more susceptible to toxins and physiologically constrained. Likewise, cells growing in a monolayer on a flat adherent substrate have drastically reduced cell-cell and cell-extracellular matrix. Herein lays the motivation to expand into the third dimension - the ability of a cell culture to accurately model molecular gradients that modulate the transport of soluble components such as oxygen and signaling molecules within the cell mass, together with aspects of the tissue organization and structure, are critical aspects of the diseased tissue and must be taken into account early on in the drug testing [6]. The native tumor environment can only be accurately recreated - or at least modeled - in 3D cell cultures [7].

Studies have shown that candidate drugs are significantly less effective in 3D compared to similar studies conducted using 3D cell cultures, a clear indication of the significant effect that the tumor microenvironment has on the response to treatment. For example, the sensitivity of OVCAR5 cells in 3D cell cultures is half of that observed in a 2D monolayer environment [8]. 3D systems more closely represents the in vivo response not only in terms of architecture and nutrient supply, but also with regard to the expression of disease biomarkers and the activity of signaling pathways [nature]. The different chemosensitivity in 2D versus 3D systems can be attributed to a number of features that arise from the tumor architecture, including the penetration of the drug through multiple layers of cells and the activation of signaling pathways which are suppressed in 2D cell cultures [5]. In 3D cell cultures, the PS cannot normally easily reach the inner most tumor cells; there is also an overexpression of hypoxia genes which depletes the oxygen concentration in the cell. This effect is even more exacerbated in PDT due to oxygen consumption [9]. 
The last years have seen the emergence of several different types of 3D cell cultures, for example tissue explants and cellular spheroids $[6,7,10]$. Especially spheroid structures are used more and more; however, a major limitation is the difficulty in scaling them up uniformly for drug screening. Additionally, they are exhibit slow spontaneous aggregation and one lacks control over their final size and shape. Likewise, the ability to study physiological signals is also limited due to the diffusion gradients in gel based systems. Nevertheless, spheroid cultures have served their purpose in opening the door to major conceptual advances [10].

These obstacles can be overcome by using self-assembling hydrogels as molecular scaffolds [9]. It allows control of size and shape, and thus tunes the molecular gradient and the oxygen concentration in the tumor model. In principle one can now alter the 3D model to fit a specific type of cancer. For example, ovarian cancer results from the attachment of single cells onto a peritoneal surface, which eventually leads to micro nodular tumors. This can be modelled by seeding cells on a Matrigel basement membrane forming 3D acini. The physical characteristics observed for this model were found to be highly varied, and thus representative of the actual tumor microenvironment - structures ranged from small clusters to large acini to caked accumulations of multiple acini, all of which resembles the heterogeneity of the disease in the body [8]. Additionally, the 'entrapment' of cells in the matrix gives more accurate data on cell death [11].

Still, this advancement has its limitations, too. For PDT drugs these relate to issues with imaging and how to monitor the overall efficacy of PDT in 3D in vitro models. Thus, development of high content screening methods can be more difficult for 3D models, but reliable protocols have emerged [8,12]. At a more fundamental level the questions arises whether different photochemical mechanisms are present in $2 \mathrm{D}$ or $3 \mathrm{D}$ in vitro and in vivo systems and how this can be utilized for the de novo design of photosensitizers $[9,13]$. Analytical problems relate to the limited ability of microscopes for visualizing complex spheroid structures requiring special quantitative imaging techniques [14]. Other PDT specific issues of 3D cultures relate to the light component of the treatment [13]. Dosimetry can be problematic, typically analysis centers on measurement of the fluence rate, which does not take the optical properties or geometry of the tumor into account [15]. Additionally, problems arise from the use of different imaging methods in different studies, which makes a comparative analysis of drug candidates from different laboratories difficult.

Nevertheless, studies from recent years indicate the validity of the "3D approach" for photosensitizer testing. These include the use of self-assembling peptides as a cellular scaffold to model drug and oxygen diffusion effects indicating that mass transfer is the main factor differentiating 2D and 3D responses [9]. 3D cultures can be used to evaluate the theranostics potential of new drugs [16] Other recent examples are the modeling of tumorstroma interactions [17], mixed neuron/cancer cell systems [11], the specific optimization of PDT drugs for hypoxic tumor environments [12], two-photon PDT studies [18], and a microfluidal breast cancer tissue model based on lab-on-a-chip technology [19], to name a few. Another noteworthy development is the use of optical coherence tomography for the visualization and study of 3D PDT models [20], which indicates the analytical and predictive power of contemporary imaging techniques in conjunction with modern tissue models.

Clearly, the realm of in vitro testing for PDT is rapidly moving into the third dimension. While all cancer drug development appears to slowly moving towards the use of $3 \mathrm{D}$ cell models the added complexity of PDT action makes this a conditio sine qua non for the screening of new photosensitizers. 


\section{Financial \& competing interests disclosure}

This work was supported by grant IVP 13/IA/1894 from Science Foundation Ireland (SFI). The authors have no other relevant affiliations or financial involvement with any organization or entity with a financial interest in or financial conflict with the subject matter or materials discussed in the manuscript.

No writing assistance was utilized in the production of this manuscript.

\section{References}

1 Dougherty TJ, Gomer CJ, Henderson BW, Jori G, Kessel D, Korbelik M, Moan J, Peng Q. Photodynamic therapy. J. Natl. Cancer Inst. 90, 889-905 (1998).

2 Castano AP, Demidova TN, Hamblin MR. Mechanisms in photodynamic therapy: part onephotosensitizers, photochemistry and cellular localization. Photodiagn. Photodyn. Ther. 1, 279-293 (2004).

3 Ethirajan M, Chen Y, Joshi P, Pandey RK. The role of porphyrin chemistry in tumor imaging and photodynamic therapy. Chem. Soc. Rev. 40 340-362 (2011).

4 Rogers L, Senge MO. The translocator protein as a potential molecular target for improved treatment efficacy in photodynamic therapy. Future Med. Chem. 6, 775-792 (2014).

5 Lee JM, Mhawech-Fauceglia P, Lee N, Parsanian LC, Lin YG, Gayther SA, Lawrenson K. A threedimensional microenvironment alters protein expression and chemosensitivity of epithelial ovarian cancer cells in vitro. Lab. Invest. 93, 528-542 (2013).

6 Pampaloni F, Reynaud EG, Stelzer EHK. The third dimension bridges the gap between cell culture and live tissue. Nature Rev. Mol. Cell Biol. 8, 839-845 (2007).

7 Baker BM, Chen CS. Deconstructing the third dimension - how 3D culture microenvironments alter cellular cues. J. Cell Sci. 125, 3015-3024 (2012).

8 Rizvi I, Celli JP, Evans CL, Abu-Yousif AO, Muzikansky A, Pogue BW, Finkelstein D, Hasan T. Synergistic enhancement of carboplatin efficacy with photodynamic therapy in a threedimensional model for micrometastatic ovarian cancer. Cancer Res. 70, 9319-9328 (2010).

9 Alemany-Ribes M, García-Díaz M, Busom M, Nonell S, Semino CE. Toward a 3D cellular model for studying in vitro the outcome of photodynamic treatments: Accounting for the effects of tissue complexity. Tissue Eng. Pt. A. 19, 1665-1674 (2013).

10 Breslin S, O'Driscoll L. Three-dimensional cell culture: the missing link in drug discovery. Drug Discov. Today 18, 240-249 (2013).

11 Wright KE, Liniker E, Loizidou M, Moore C, MacRobert AJ, Phillips JB. Peripheral neural cell sensitivity to $\mathrm{mTHPC}$-mediated photodynamic therapy in a 3D in vitro model. Br. J. Cancer. 101, 658-665 (2009).

12 Klein OJ, Bhayana B, Park YJ, Evans CL. In vitro optimization of EtNBS-PDT against hypoxic tumor environments with a tiered, high-content, 3D model optical screening platform. Mol. Pharmaceutics 9, 3171-3182 (2012).

13 Salas-García I, Fanjul-Vélez F, Arce-Diego JL. Superficial radially resolved fluorescence and 3D photochemical time-dependent model for photodynamic therapy. Opt. Lett. 39, 1845-1848 (2014).

14 Celli CP, Rizvi I, Evans CL, Abu-Yousif AOA, Hasan T. Quantitative imaging reveals heterogeneous growth dynamics and treatment-dependent residual tumor distributions in a three-dimensional ovarian cancer model. J. Biomed. Opt. 15, 051603 (2010).

15 Rizvi I, Anbil S, Alagic N, Celli JP, Zheng LZ, Palanisami A, Glidden MD, Pogue BW, Hasan T. PDT Dose Parameters Impact Tumoricidal Durability and Cell Death Pathways in a 3D Ovarian Cancer Model. Photochem. Photobiol. 89, 942-952 (2013). 


\section{EdITORIAL | SENGE AND STAFFORD}

16 Glidden MD, Celli JP, Massodi I, Rizvi I, Pogue BW, Hasan T. Image-Based Quantification of Benzoporphyrin Derivative Uptake, Localization, and Photobleaching in 3D Tumor Models, for Optimization of PDT Parameters. Theranostics 2, 827-839 (2012).

17 Celli JP. Stromal interactions as regulators of tumor growth and therapeutic response: A potential target for photodynamic therapy? Isr. J. Chem. 52, 757-766 (2012).

18 Liu J, Li G, Zhang P, Jin C, Zeng L, Ji L, Chao H. Ruthenium(II) polypyridyl complexes as mitochondria-targeted two-photon photodynamic anticancer agents. Biomaterials 56, 140153 (2015).

19 Yang $\mathrm{Y}$, Yang $\mathrm{X}$, Zou J, Jia C, Hu Y, Du H, Wang H. Evaluation of photodynamic therapy efficiency using an in vitro three-dimensional microfluidic breast cancer tissue model. Lab. Chip. 15, 725-744 (2015).

20 Jung Y, Nichols AJ, Klein OJ, Roussakis E, Evans CL. Label-Free, Longitudinal Visualization of PDT Response In Vitro with Optical Coherence Tomography. Isr. J. Chem. 52, 728-744 (2012).

Author photo: Mathias O. Senge

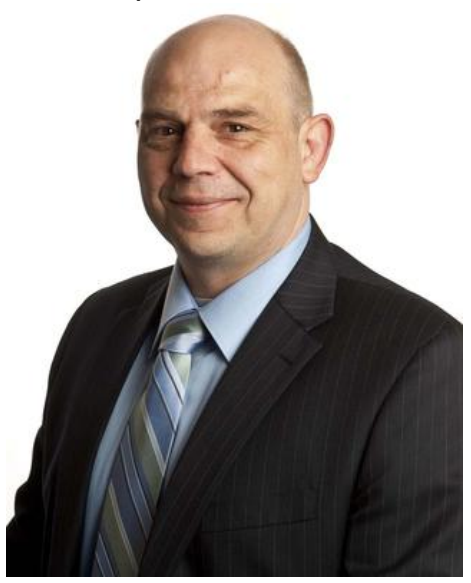

Author photo: Shelley Stafford

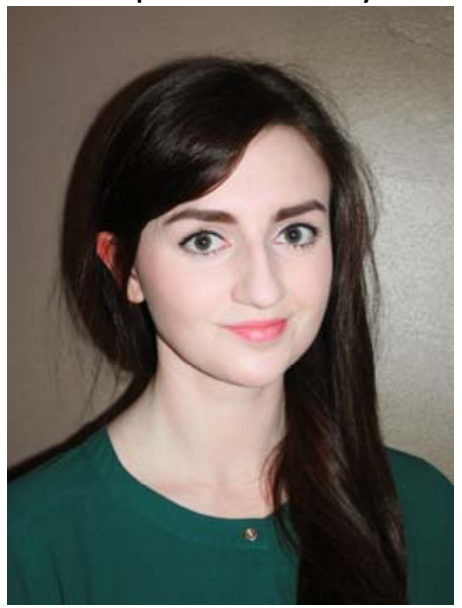

